\title{
Analysis on the Penetration of Emotional Education in College Physical Education Based on Emotional Feature Clustering
}

\author{
Hong Guo' and Miqi Wang $\mathbb{D}^{2}$ \\ ${ }^{1}$ Department of Public Physical Education, Hebei Normal University, Shijiazhuang, Hebei 050024, China \\ ${ }^{2}$ Sports Department, Guangdong University of Technology, Guangzhou, Guangdong 510006, China \\ Correspondence should be addressed to Miqi Wang; wmq_tyb@gdut.edu.cn
}

Received 18 December 2021; Revised 2 January 2022; Accepted 3 January 2022; Published 14 February 2022

Academic Editor: Tongguang Ni

Copyright (c) 2022 Hong Guo and Miqi Wang. This is an open access article distributed under the Creative Commons Attribution License, which permits unrestricted use, distribution, and reproduction in any medium, provided the original work is properly cited.

\begin{abstract}
Physical education is a highly skilled education offered in colleges and universities. Teachers do not appear in front of inanimate machines as laborers, and they are not the same as gardeners who grow colorful trees, according to their essential characteristics. Their work is aimed at flesh-and-blood students who are sentimental, thoughtful, and engaged in critical thinking. As a result, schools should prioritize physical education and place a premium on emotional infiltration education, improve learning interest, improve teacher-student relationships, create a harmonious teaching environment, and improve teaching quality; it has a significant impact on an individual's entire life. The modern educational process places a premium on the transmission of rational knowledge while overlooking the accumulation of emotional experience. The cultivation and development of emotional feeling ability, emotional expression, and expression ability receive less attention than the training and improvement of language, concept, logic, and reasoning abilities. Emotion feature clustering is used to propose an emotion recognition method in this study. This method generates extended features for classification by constructing a co-occurrence matrix based on the co-occurrence relationship of emotion features and then by applying the spectral clustering method. The binary value of whether emotional features of emotional education in college physical education appear in a particular cluster is then expressed as a feature and extended to the original training feature set, alleviating the problem of sparse features.
\end{abstract}

\section{Introduction}

In contemporary universities, there will be such a situation, that is, students who are active in the playground will be stronger than those who lack physical exercise, which has to cause concern in schools. Physical education in colleges and universities is a highly skilled education. Its essential characteristics determine that teachers do not appear in front of inanimate machines as laborers and are different from gardeners who cultivate colorful trees. Their work targets young students who are full of flesh and blood, sentient, thoughtful, and active in thinking. This determines that schools should play a leading role in physical education and attach importance to and implement emotional infiltration education [1]. According to modern psychology theory, the factors that affect students' learning include not only their intelligence factors but also nonintelligence factors such as emotion and will. This nonintelligence factor can lay a good psychological foundation for a person's socialization and meet his psychological needs [2]. In the past, physical education in colleges and universities mainly focused on competitive minority sports, but now our requirements should develop into popular sports of art and ecological sports so that sports can become a necessity for people to survive. Therefore, in college physical education, physical education teachers should add emotional education instead of simply teaching physical education [3], improve interest in learning, harmonize the relationship between teachers and students, form a harmonious teaching atmosphere, and improve teaching quality, which even has an important influence on his whole life. The modern educational process emphasizes 
the imparting of rational knowledge and neglects the accumulation of emotional experience. Emphasis is placed on the training and improvement of language, concept, logic, and reasoning ability but less on the training and development of emotional feeling ability, emotional expression, and expression ability [4,5]. This educational model ignores people's emotional characteristics, cannot meet people's different emotional needs, cannot stimulate and regulate people's emotional mechanism, and cannot say it has a profound impact on people's emotional aspects.

The method of emotion recognition proposed in this study is based on emotion feature clustering. This method generates extended features for classification by constructing a co-occurrence matrix based on the co-occurrence relationship of emotion features and then by using the spectral clustering method. Because the training dataset for the learning method of emotional feature clustering is small, there may be an emotional education situation in college physical education where the features in the test dataset do not appear in the training dataset, resulting in unsatisfactory classification results $[6,7]$. To put it another way, the sparsity of this feature distribution has an impact on emotion classification accuracy. To complete the final emotion classification, the two classifiers collaborate. The method proposed in this study is based on the following basic assumption: in college physical education with similar emotional tendencies, emotional words of emotional education have a high probability of appearance in consumer comments with similar emotional tendencies; these "similar" emotional features can then be clustered based on their cooccurrence relationship in comments $[8,9]$. The goal of image retrieval based on emotional semantics is to investigate the emotional analysis of emotional education features in college physical education, to describe and extract these features in a way that is appropriate for people's psychological reactions to the features, and to cluster the emotional features [10].

The emotion recognition algorithm based on emotion feature clustering is based on an observation: words or phrases of emotional education in college physical education with the same emotional tendency are more likely to appear in the same product comments $[11,12]$. For example, in general, the probability of "very good" and "perfect" appearing in the same comment with a favorable emotional tendency is higher than that of "very poor" and "perfect" appearing in a comment together [13]. By using the emotional characteristics of emotional education in college physical education with unlabeled emotional category data to cluster the emotional characteristics, the number of available clusters is limited [14]. Then, at this time, the binary value of whether the emotional features of emotional education in college physical education appear in a certain cluster is expressed as a feature and extended to the original training feature set, which can alleviate the problem of sparse features. Emotion recognition algorithm does not depend on the specific mathematical description, has good global complex combination optimization ability, and has penetrated into many fields, such as coloring problem, vehicle route planning problem, and so on [15].

\section{Related Work}

Literature [16] puts forward that in college physical education, emotional educators enthusiastically carry out educational activities, induce and stimulate the positive emotion and positive attitude of the educatees in the process of education, make them in the best state of psychology, and regard the emotional cultivation of the educatees as one of the objectives of education. In literature [17] through the big data analysis method, in college physical education teaching, if physical education teachers can grasp students' inner feelings and use their own cultivation to adjust students' psychological trends and inner needs, they will shorten the psychological distance with students, produce a good classroom atmosphere, and achieve the desired teaching effect. Literature [18] shows that in order to implement emotional education in college physical education, teachers are required to invest in teaching with positive and full enthusiasm and good personal image; second, we should be good at using various methods and means to induce, motivate, and coordinate students' emotions. Literature [19] proposed that foreign research on text emotion recognition began in the 1990s. In text emotion analysis, researchers found two very interesting phenomena: first, emotional words with opposite tendencies generally do not appear together; second, emotional words with the same tendency often appear at the same time. In literature [20] through the big data analysis method, the professional norms of physical education teachers are highly intuitive, and their words, deeds, and emotions are very easy to have an impact on students. Literature [21] shows that the biggest feature of physical education teaching is to pay attention to physical activities. Physical education teachers' treatment methods and means of various classroom behaviors reflect their own educational wit and professional cultivation. Literature [22] puts forward that the semantic inclination of each sentence in the text can be obtained by weight first calculation method, emotional education is discussed on the combination of emotional words in college physical education, and the concept of the headword is put forward to calculate the inclination of words, which lays a foundation for more complicated emotional analysis of the text. In literature [23] through the big data analysis method, physical education teachers in colleges and universities should make full use of the advantages of disciplines in emotional education, pay attention to active and healthy emotional communication with students, win the respect and cooperation of students, and obtain the best educational effect. Literature [24] shows that the grades of praise and disapproval in college physical education can be divided into three categories (positive emotion, negative emotion, and neutral emotion) by star rating index, and the polarity classification of emotional education in comment text is completed by using the experimental algorithm using three classification methods, among which the method of the support-vector machine gets higher accuracy. Literature [25] puts forward a method 
of semantic correlation field based on two semantic tendencies of emotional word dictionary as the calculation basis of emotion classification.

Emotional education in college physical education is investigated in this study. Based on an emotional feature clustering algorithm, words or phrases with similar emotional connotations are more likely to appear in the same product review. In general, the likelihood of "very good" and "perfect" appearing in the same comment with a commendatory emotional tendency is higher than the likelihood of "very poor" and "perfect" appearing in the same comment with a disparaging emotional tendency. This study believes that if we can cluster these emotional characteristics based on their co-occurrence relationship, it will be beneficial to emotional education in college physical education.

\section{Emotion Recognition Algorithm and Model Based on Emotion Feature Clustering}

The emotion recognition algorithm of emotion feature clustering needs to label the data manually, which may require a lot of manpower and time. This is the most difficult and challenging work in the emotion recognition algorithm system. This study uses emotion feature clustering college physical education as the research object and considers the influence of different weights of interested and noninterested regions of emotional education on feature extraction. However, how to make users interactively select eigenvalues and set the weight of eigenvalues is a problem worthy of discussion. The emotion classification method of emotion recognition algorithm based on emotion feature clustering only needs to manually label a small amount of training data and then uses a large number of data without labeling the categories of emotion to train the learner, so as to classify consumer comments of unknown categories of emotion. This study selects 600 pictures in emotional education in college physical education as the original dataset, that is, the research object. The emotion analysis steps of analyzing emotion feature clustering are shown in Figure 1.

There is only a small amount of training data in the training dataset of emotion analysis of emotion recognition algorithm, so it may happen that the features in the test dataset do not appear in the training dataset, which may lead to unsatisfactory classification results. That is to say, the sparsity of feature distribution will affect the accuracy of emotion analysis. Emotional feature clustering based on the partition is the most widely used clustering. Its purpose is to divide the dataset into several subsets, that is, given a dataset with $n$ tuples or records, $k$ groups are constructed, and each group represents a cluster (for a given $k$, we can give an initial grouping method and then change the grouping through repeated iterations, so that the grouping scheme after each improvement is better than the previous one). This study describes the basic block diagram of the emotion recognition algorithm with divided emotion features, in which there are various methods in the first three steps, and different emotion recognition algorithms with divided emotion features can be obtained through combination, as shown in Figure 2.
The partition method is to divide $n$ objects in set $u$ into a given $K$ subset so that the object similarity in the same set is high, while the object similarity in different sets is low. The definition of similarity is the key to division. Generally, it defines the objective function in the form of a formula and uses the heuristic method to minimize the value of the objective function.

$$
J=\frac{1}{N} \sum_{K=1}^{K} \sum_{n=1}^{N} u_{n k} d^{2}\left(\bar{x}_{k}-x_{n}\right),
$$

where $j$ is the sum of the mean square deviations of all objects in the set $\mathrm{U}$ and the corresponding cluster centers, $\mathrm{xn}$ is a data object in the space representing the objects, and $x_{k}$ is the mean value of the cluster $C_{k}$. The formula of clustering standard aims to make the obtained clusters have the following characteristics: each cluster itself is as compact as possible, and each cluster is separated as much as possible.

The basic idea of emotion analysis based on emotion feature clustering and emotion recognition algorithm is briefly introduced: data are regarded as emotion analysis with different attributes, and the cluster center is the "emotion source" that emotion analysis is looking for, so the data clustering process can be regarded as the process of emotion analysis looking for emotion. It is assumed that the data object is $x=\{\mathrm{xi} \mid \mathrm{xi}=(\mathrm{xi} 1, \mathrm{xi} 2, \ldots, \mathrm{xim}), I=1,2,3, \ldots, n\}$. First, the algorithm is initialized. The pheromone $\tau(\mathrm{O})=0$ of each path is set to $O$, and the parameters such as cluster radius $R$, statistical error $\varepsilon$, and representative object $M$ are set. The weighted Euclidean distance dij between objects Xi and $\mathrm{Xj}$ is calculated, and the pheromones on each route are calculated as follows:

$$
\tau_{i j}(t)=\left\{\left(\begin{array}{l}
1, d_{i j} \leq r \\
0, d_{i j}>r
\end{array}\right) .\right.
$$

The probability formula for merging objects $\mathrm{Xi}$ into $\mathrm{XJ}$ is as follows:

$$
P_{i j}=\frac{\tau^{a} i j(t) \eta^{\beta} i j(t)}{\sum_{s c S} \tau^{a} s j(t) \eta^{\beta} i j(t)},
$$

where $S=\left\{x_{s} \mid d_{s j} \leq R, s=1,2, \ldots, J, j+1, \ldots, n\right\}$. If PIJ $(T)$ is greater than the threshold $P_{0}, X_{i}$ is merged into the domain of $X_{J}$. Among the above clustering methods, the representative object $m$ has a great impact on the operation efficiency and clustering results, and there are many selection methods of representative objects. Different methods can be tried according to the situation to avoid the algorithm falling into local optimization. Although the number of clusters does not need to be given in advance, the scale of clustering is limited because the cluster radius is preset.

The cluster centers of these two clusters are calculated according to the following formula, where $n_{1}$ is the number of samples in cluster $\mathrm{A} 1$ and $\mathrm{n} 2$ is the number of samples in cluster A2.

$$
\bar{A}=\frac{1}{n_{1}} \sum_{k=1}^{n_{1}} X_{k}\left(X_{k} \in A_{i}\right)(i=1,2) .
$$




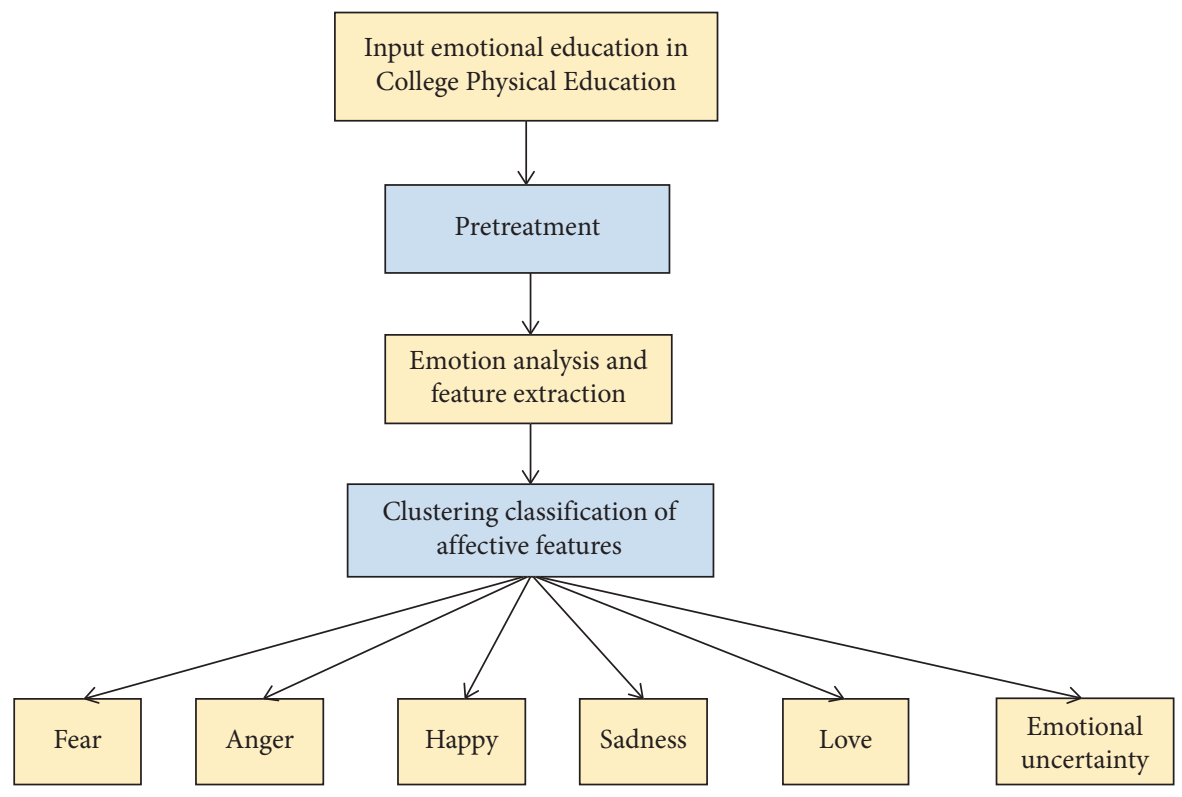

Figure 1: Flowchart of emotion feature clustering emotion recognition algorithm.

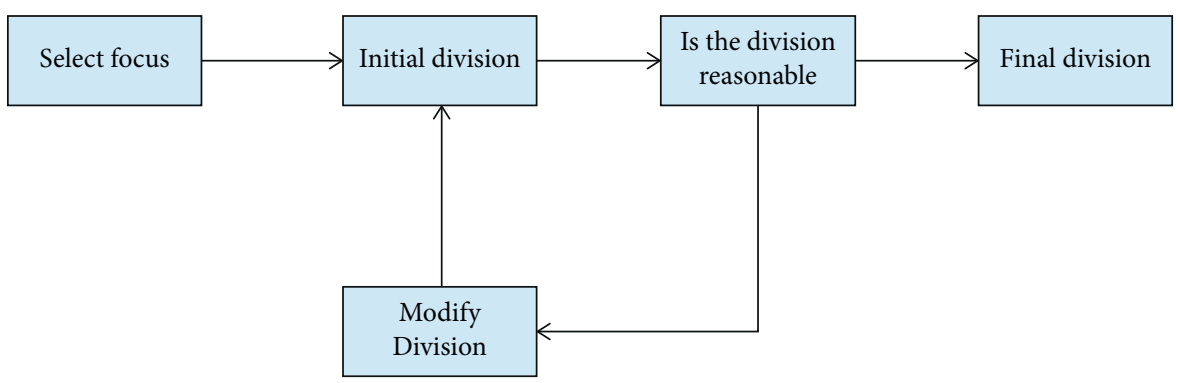

FIGURE 2: Framework of emotion recognition algorithm for emotion feature clustering.

After clustering, the distance between any two adjacent classes is calculated according to the clustering center of each class. If the distance between classes is less than the threshold value $\varepsilon$, the two categories are merged and the cluster center is updated at the same time. The distance between two classes is defined as the distance between the nearest two samples $X$ and $Y$ in two classes $s$ and $V$.

$$
d(S, V)=\min (d(x, y), x \in S, y \in V) .
$$

If we use emotion feature clustering in unlabeled emotion category data to cluster emotion features, the number of available clusters of emotion recognition algorithms is limited. The presence of an emotion feature cluster in the cluster of an emotion recognition algorithm is then expressed as a feature and extended to the original training feature set, alleviating the problem of feature sparseness.

Emotional feature clustering is a model that considers the probability relationship between physical and emotional education before and after college. It depicts the text's word sequence relationship. As a result, it is widely used in a variety of emotional recognition problems, including text word segmentation, automatic input, part of speech tagging, machine translation, and so on. In simple terms, the emotion feature clustering emotion recognition algorithm model counts the likelihood of a sentence or word sequence.

$$
P\left(W_{1}, W_{2}, \ldots W_{K}\right),
$$

where $\mathrm{W}$ represents each word, and $k$ represents the length of the word sequence. We borrow the knowledge of the conditional probability of probability theory.

$$
\begin{aligned}
& P\left(W_{1}, W_{2}, \ldots, W_{K}\right) \\
& \quad=P\left(W_{1}\right) P\left(W_{2} \mid W_{1}\right) \ldots P\left(W_{K} \mid W_{1}, W_{2}, \ldots, W_{K-1}\right) .
\end{aligned}
$$

But if the length of $k$ is too long, the word sequence modeling needs a very complicated model. Therefore, in engineering implementation, an approximation is often needed. Common methods include the n-gram model, maximum entropy model, conditional random field model, neural network model, and so on.

Affective feature clustering is one of the most used statistical affective feature clustering affective recognition algorithm models. The n-gram model is also known as the 
n-1-order Markov model. It is based on Markov's conditional hypothesis, that is, the probability of the current word is only related to the probability of the previous n-1 word.

$P\left(W_{K} \mid W_{1}, W_{2}, \ldots, W_{K-1}\right) \approx P\left(W_{K} \mid W_{K-N+1}, \ldots, W_{K-1}\right)$.

Therefore, the probability of word sequence is approximately

$$
P\left(W_{1}, W_{2}, \ldots, W_{K}\right) \approx \prod_{l=1}^{K} P\left(W_{1} \mid W_{l-n+1}, \ldots, W_{l-1}\right) .
$$

When the value of $n$ is 1,2 , and 3, the corresponding emotion feature clustering emotion recognition algorithm models are called unigram, bigram, and trigram models, respectively. With the increase in grammar order, the model is more complex, so it is necessary to select the appropriate order in a specific application. The parameter estimation of the n-gram model generally uses maximum likelihood estimation (MLE).

$$
P\left(W_{l} \mid W_{L-N+1}, \ldots, W_{l-1}\right)=\frac{\operatorname{Cont}\left(W_{l-n+1}, \ldots, W_{1}\right)}{\operatorname{Count}\left(W_{L-n+1}, \ldots, W_{l-1}\right)} \text {. }
$$

\section{Application of Emotional Education in College Physical Education Based on Characteristic Clustering to Development}

4.1. Emotional Education in College Physical Education Based on Emotional Feature Clustering. The emotion recognition algorithm of feature clustering is the sensory experience and reaction of people to emotional education in college physical education and the psychological stress state caused by the stimulation of external things to people's brains. Generally speaking, emotions include positive and negative, and each of them may contain rich content. In many cases, they are connected, and the community is now in people's reactions to external things. Physical education teachers and students are two sources of emotional analysis in physical education teaching. The greatest feature of physical education is to attach importance to physical activities, and the ways and means of dealing with various classroom behaviors by physical education teachers reflect their own educational wit and professional accomplishment. In the process of physical education, infiltrating the necessary emotional education can help college students learn respect, tolerance, friendliness, and self-confidence, cultivate collectivism and perseverance, and contribute to the formation of college students' good personalities. Any sport can show the strength, speed, dexterity, and vitality of human body and show the beauty of form and soul. Infiltration of emotion recognition education in physical education can help college students understand and appreciate this beauty, broaden their aesthetic vision, and promote their esthetic pursuit, thus forming a good esthetic taste. The emotion recognition algorithm of feature clustering is applied to emotional education in physical education in colleges and universities. Combined with the research results of modern educational information technology, volleyball teaching theory and methods and other aspects of emotion recognition algorithm, theoretical analysis, and experimental research are carried out in order to solve the one-sidedness of conventional teaching to cultivate students and further explore the influence of this emotion analysis on students' learning interest and academic achievement. Physical education teaching in colleges and universities is mostly in a relaxed environment, and many routine sports events are included in the teaching content, which broadens the vision of college students' sports knowledge, enriches their spare-time activities, and is conducive to stimulating and cultivating college students' emotional analysis of novelty, happiness, and satisfaction.

Physical education teaching characteristics determine that college students can gain a wide range of emotional analysis experience. For example, students will feel satisfied and happy after learning and mastering the fundamentals of sports movements; tension will arise during sports tests and competitive competitions; if you pass the test without difficulty or achieve good results in the competition, you will feel happy; and so on. Physical education teachers' professional norms are highly intuitive, and their words, actions, and emotions have a significant impact on their students. Individuals and classes receive physical education together in the process of physical education in colleges and universities, and physical activities necessitate competition. They often require mutual encouragement and assistance when competing as a team. They must learn to observe, unite, and love one another. It is simple to improve college students' collective reputation and friendship among peers, which is conducive to the development of a harmonious teacher-student and classmate relationship. The sports psychology, sports skills, and acceptance ability of students in college physical education differ. As educators, they should treat each student with respect and understanding, set different goals for them, use different methods and means to promote their progress, and encourage them to build confidence. As a result, physical education teachers should make full use of the subject's advantages in emotional education, pay special attention to positive and healthy emotional analysis, communication, and communication with students, earn students' respect and cooperation, and achieve the best educational effect possible. "Rational education" is the polar opposite of emotional learning.

This kind of education does not include emotional analysis into the feasible means of education. The emotional identification algorithm in emotional feature clustering regards the goal of knowledge imparting as the central position of emotional education, which is manifested in the indifference, distortion, and obstruction of students' emotional needs in the process of emotional education, the lack of normal emotional analysis and communication between teachers and students, the lack of vitality in emotional education activities, the inflexible form of emotional education, and the lack of initiative and interest of students. In the practice of physical education teaching in colleges and universities, college students need to bear certain sports load and overcome different degrees of difficulties. In this 


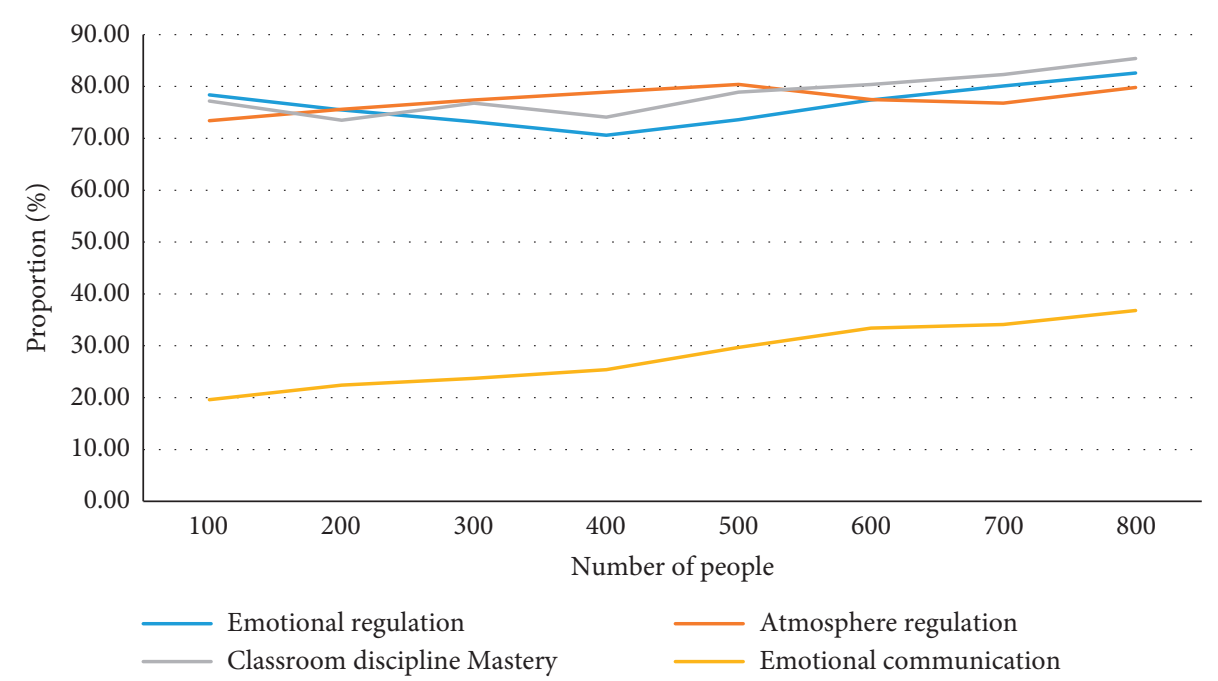

Figure 3: Proportion chart of emotional communication in college physical education.

process, college students can realize their own values and get different degrees of satisfaction, which will effectively enhance students' collective sense of honor. In college physical education teaching, we should not only cultivate students' action skills but also organize practice methods and logical thinking ability. It is also necessary to train students to have good learning psychological quality, personality quality, and esthetic quality, which are all indispensable and important components in professional emotion teaching. However, in the traditional teaching quality, action technology occupies the absolute center, and the factors of ability training are not given due attention, which has caused various defects and set up many obstacles to the implementation of quality education. Students have all kinds of emotional analysis experiences in their studies. Teachers must go deep into them and communicate with them in order to implement teaching more accurately. This is the emotional communication function of emotional education in physical education. As an activity requiring collective participation, the physical education class's teaching nature determines that students need to help and cooperate with each other in the course of class. During training, students are both practitioners and protectors of classmates, which can effectively cultivate students' sense of responsibility and obligation. A person's emotional experience is closely related to his life attitude, mental state, work efficiency, and intellectual potential, and it is an important part of his intelligence, personality, and thinking development. Emotion clustering is the driving force of the emotion recognition algorithm to catalyze college students' cognitive understanding, promote their moral level, and motivate students to make progress.

4.2. Analysis of Experimental Results. Physical education teachers should make full use of the advantages of the subject in emotional education, pay attention to positive and healthy emotional analysis, communication, and communication with students, win students' respect and cooperation, and obtain the best educational effect. The author conducted a questionnaire survey on the classroom behavior and influence of PE teachers on 600 college students of 2019 and 2020 in a university and made a statistical analysis, as shown in Figure 3.

One of them is emotional communication. Nearly $80 \%$ of students think that teachers do not communicate with them emotionally, there is emotional distance between teachers and students, and students stay away from teachers, which directly affect the teaching effect. Students have all kinds of emotional experiences in their studies. Teachers must go deep into them and communicate with them in order to implement teaching more accurately. This is the emotional communication function of emotional education in physical education, as shown in Figures 4-6.

Figure 4 shows that after the experiment, the experimental and control classes' interest in physical education emotional education showed significant differences, indicating that incorporating emotional teaching theory into the professional course of physical education emotional education in colleges and universities can improve students' interest in physical education. The reason for this is that applying emotional analysis teaching theory to professional physical education courses in colleges and universities meets students' direct physical education needs and stimulates their desire for sports. Students' direct sports needs refer to a desire to learn about or participate in a sport because it interests them. Figure 5 shows that the experimental and control classes significantly improved their technical evaluation results after the experiment when compared to the control class, and the difference is significant. It demonstrates that using emotional education theory in physical education classes is more conducive to mastering technical skills and improving the teaching effect of emotional analysis in physical education classes than traditional classes.

As can be seen from the statistical results in Figure 6, there is no significant difference between the students in the experimental class and the control class after the experiment. This shows that emotional teaching and conventional teaching methods in volleyball major courses in colleges and 


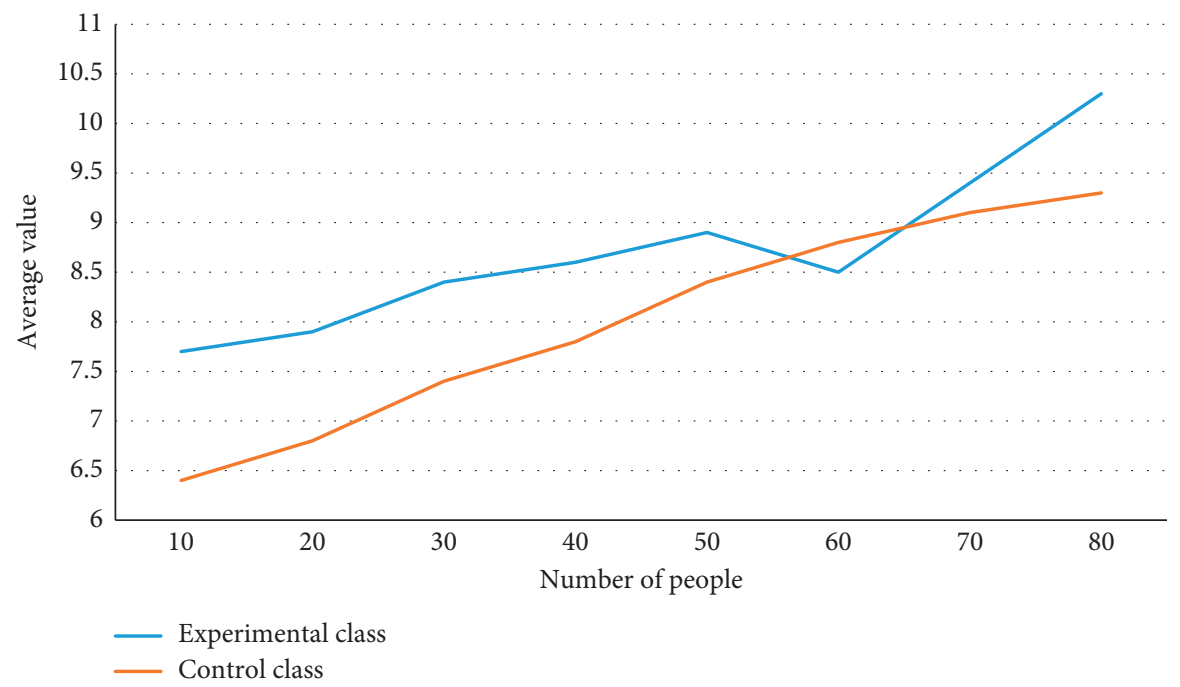

FIgURE 4: Comparison and analysis of comprehensive technical evaluation of emotional education technology in physical education (volleyball players).

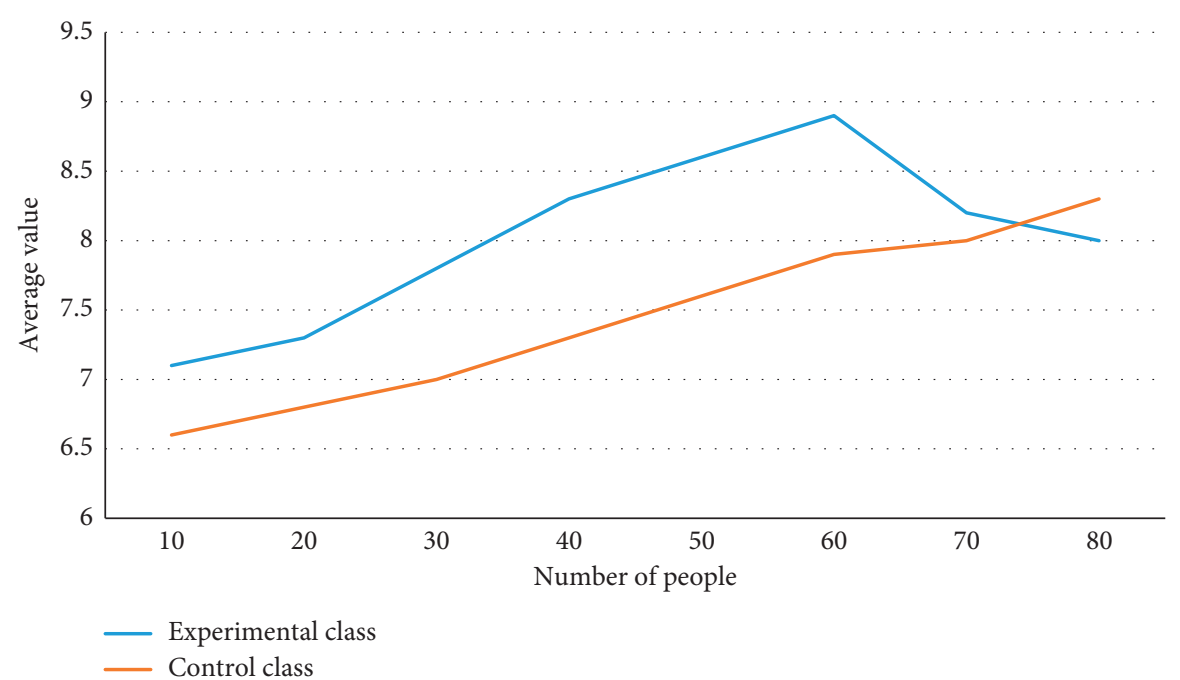

Figure 5: Comparison and analysis of comprehensive technical evaluation of emotional education technology in physical education (basketball players).

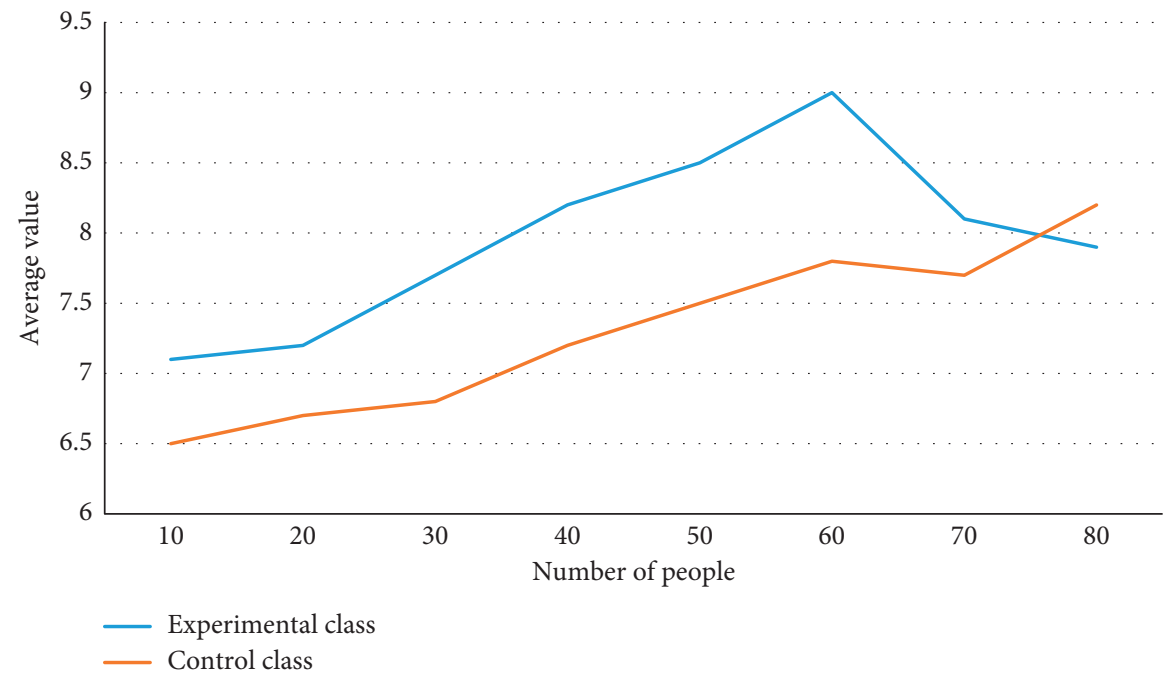

FIGURE 6: Comparison and analysis of comprehensive technical evaluation of emotional education technology in physical education (sprinters). 


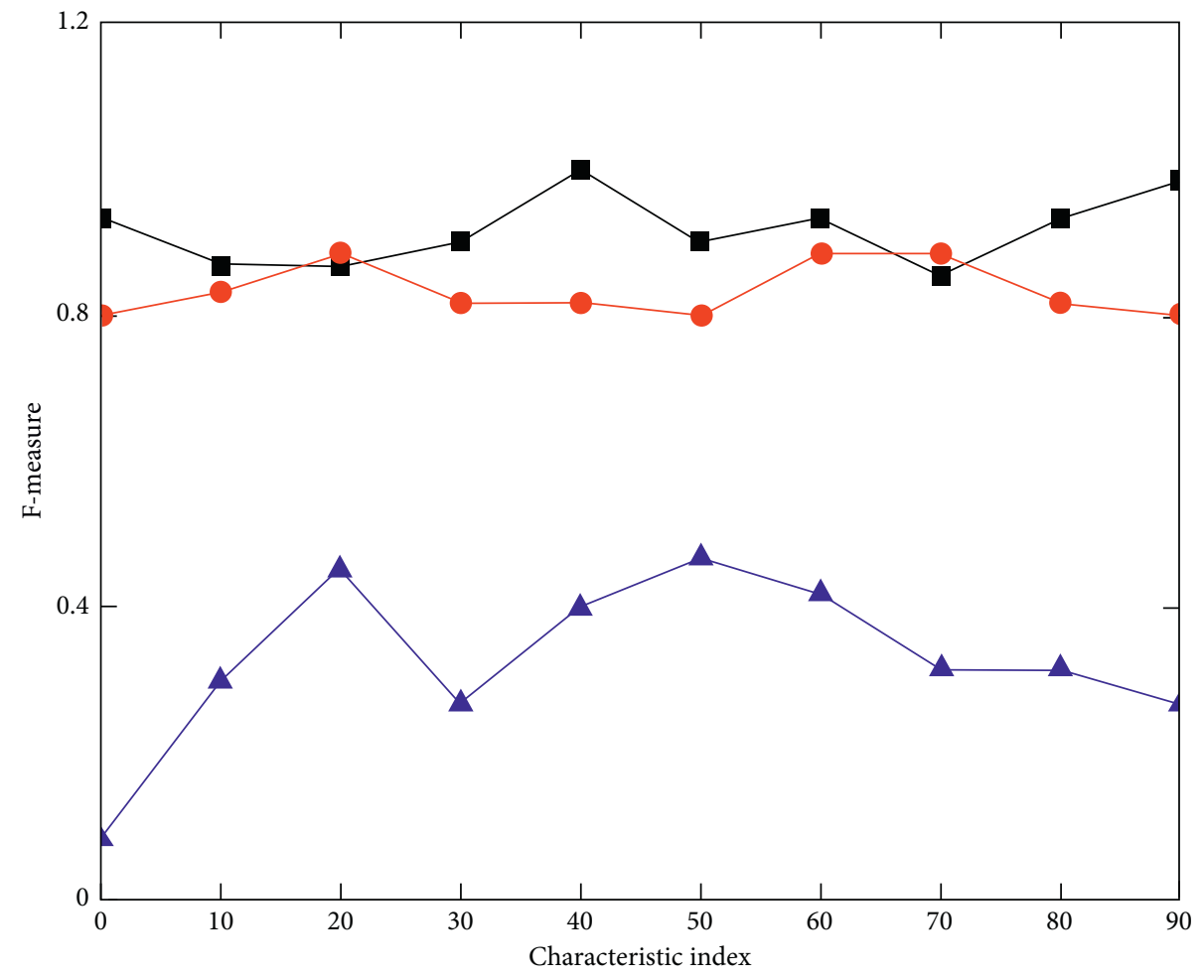

- Affective feature clustering

- NB

$\rightarrow$ SVM

FIgURE 7: Recognition effect after emotional feature clustering (volleyball corpus).

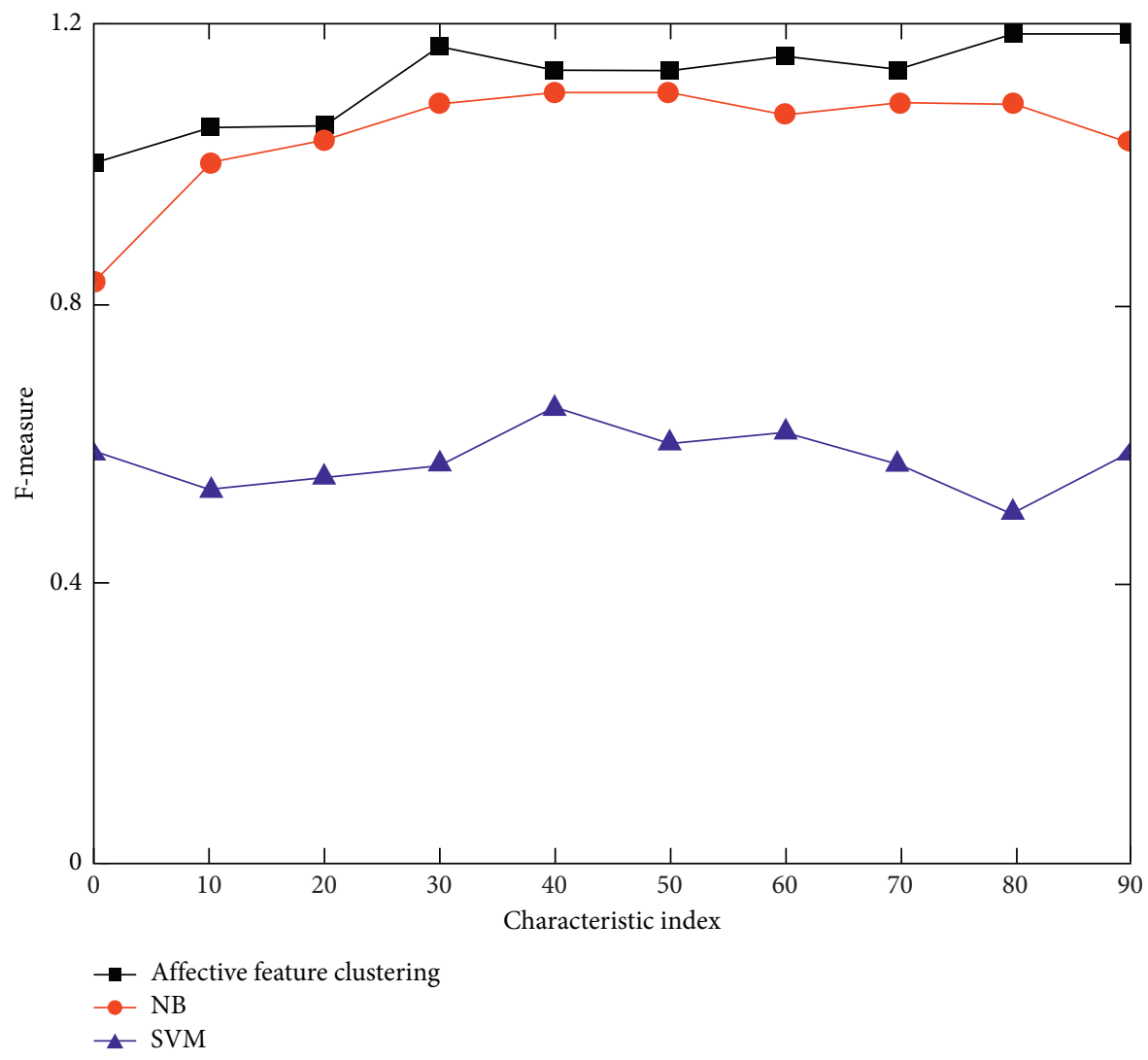

Figure 8: Recognition effect after emotional feature clustering (basketball corpus). 


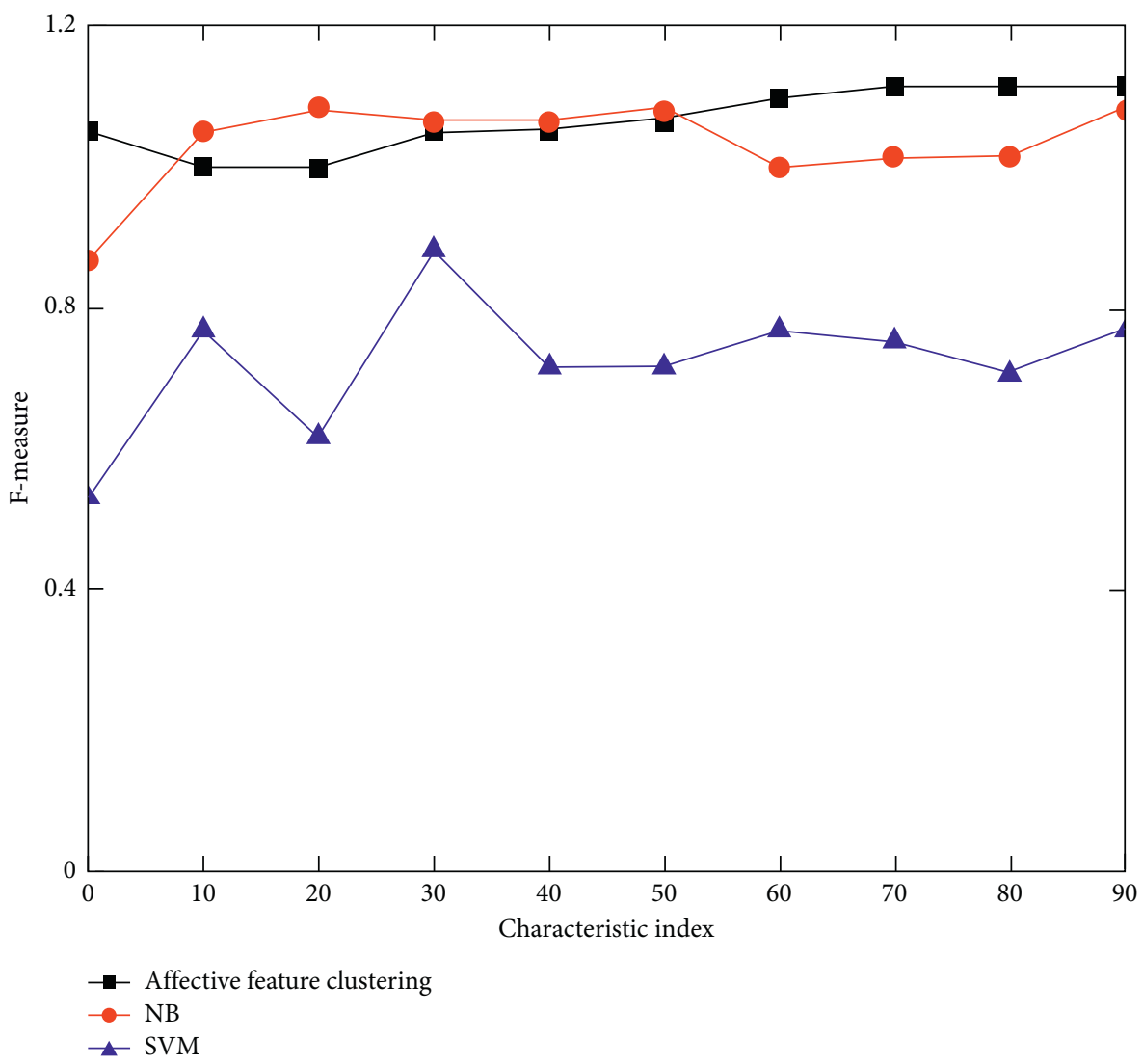

FIgURE 9: Recognition effect after emotional feature clustering (sprint corpus).

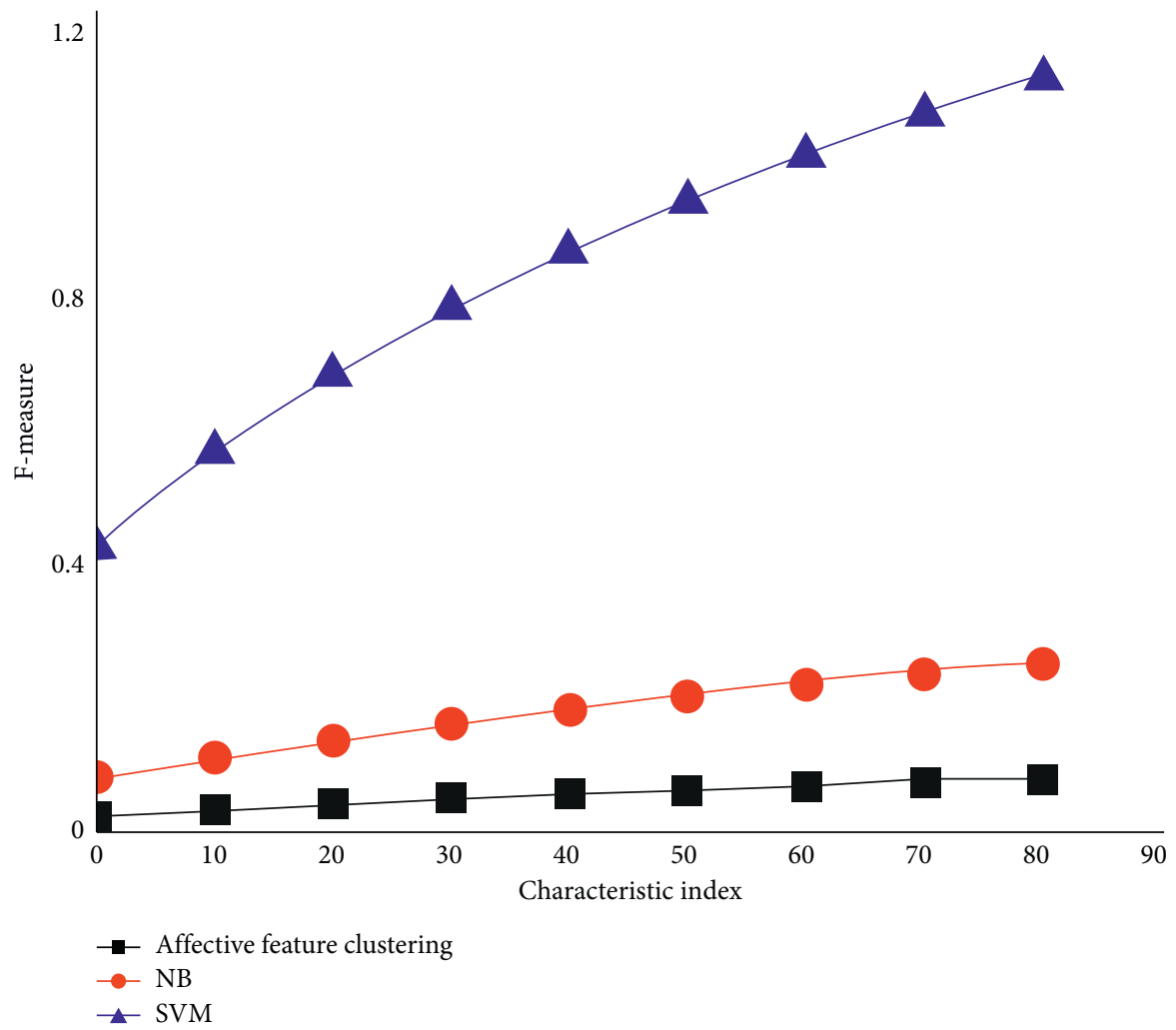

FIGURE 10: Recognition effect after emotional feature clustering (table tennis corpus). 
universities have improved students' achievement to a certain extent. Compared with conventional teaching, emotional teaching does not significantly improve students' achievement in reaching the standard because the formation of motor skills is a long-term systematic and scientific learning and training process, and the level of students' achievement in reaching the standard is related to training time and training level. After we get the word vector, we use the similar characteristics of emotional feature clustering in emotional education in college physical education. We first use the emotional recognition method to divide all words into 500 categories, then use various labels to replace the corresponding words, and then classify the text emotional analysis. About $30 \%$ of the data are used as test data and the rest as training data. The classification results are shown in Figures 7-10.

Experimental results show that SVM still has a good classification performance. In the emotional education of physical education, the mixed feature classification of ternary grammar and TFIDF has the best effect. However, compared with the recognition rate in chapter 3 , the overall recognition rate of text sentiment analysis using sentiment feature clustering results has declined. By analyzing the reasons, the biggest problem is that although Word2Vec quantifies the word direction and has good clustering characteristics, the use of clustering brings side effects of classification results: the fewer categories of emotional education in physical education in clustering will lead to the less accurate differentiation of features, for example, two words with far distance in the same category of emotional education in physical education may have more differences in meaning. Experiments have verified our conjecture, and our emotion recognition algorithm that expands the clustering category of emotional features will improve the success rate of emotional education in physical education. An extreme idea is that when each word is divided into a separate category, the classification results will be consistent with those in Section 3, that is, Word2Vec. Although the recognition rate of emotional education in physical education is not better, it also proves that the language model features and SVM classification method have higher recognition rates, and the network structure of emotional feature clustering also inspires us to use a neural network.

\section{Conclusions}

To summarize, physical education teaching characteristics determine that college students can gain a variety of emotional analysis experiences. For example, students will feel satisfied and happy after learning and mastering the fundamentals of sports movements; tension will arise during sports tests and competitive competitions; if you pass the test smoothly or achieve good results in the competition, you will feel happy; and so on. We focus on the implementation of formative evaluation in emotional teaching evaluation so that problems in the teaching process are brought to the attention of teachers and students right away, problems in teaching are remedied quickly, and students' learning errors are reduced. This study investigates emotional education in the context of college physical education. Based on emotional feature clustering, this study proposes improved methods for text features and classification algorithms using the original structure model. Before each clustering, the emotion recognition algorithm uses emotion feature clustering to calculate the two points with the smallest similarity as the starting cluster center. After clustering, the distance between each class is calculated, and the two classes with the shortest distance between them are combined. The goal of knowledge transfer is placed at the center of emotional education by the emotion recognition algorithm in emotion feature clustering. There is a dearth of standard emotion analysis and communication between teachers and students. Emotional education activities are devoid of vitality. Students lack initiative and interest as a result of the rigid nature of emotional education.

\section{Data Availability}

The data used to support the findings of this study are included within the article.

\section{Conflicts of Interest}

The authors declare that there are no conflicts of interest.

\section{References}

[1] H. Boubenna and D. Lee, "Image-based emotion recognition using evolutionary algorithms," Biologically Inspired Cognitive Architectures, vol. 24, pp. 70-76, 2018.

[2] R. Jenke, A. Peer, and M. Buss, "Feature extraction and selection for emotion recognition from EEG," IEEE Transactions on Affective Computing, vol. 5, no. 3, pp. 327-339, 2017.

[3] D. Xu, J. Zhang, H. Xu et al., "Multi-scale supervised clustering-based feature selection for tumor classification and identification of biomarkers and targets on genomic data," BMC Genomics, vol. 21, no. 1, pp. 650-654, 2020.

[4] G. Yu, "Emotion monitoring for preschool children based on face recognition and emotion recognition algorithms," Complexity, vol. 2021, no. 5, 12 pages, Article ID 6654455, 2021.

[5] J. Han, Z. Zhang, G. Keren, and B. Schuller, "Emotion recognition in speech with latent discriminative representations learning," Acta Acustica United with Acustica, vol. 104, no. 5, pp. 737-740, 2018.

[6] Y. Shi, Z. Lv, N. Bi, and C. Zhang, "An improved SIFT algorithm for robust emotion recognition under various face poses and illuminations," Neural Computing \& Applications, vol. 32, no. 13, pp. 9267-9281, 2020.

[7] T. Udhan and S. Bernadin, "Speaker-dependent low-level acoustic feature extraction for emotion recognition," Journal of the Acoustical Society of America, vol. 143, no. 3, p. 1747, 2018.

[8] B. Sun, L. Li, X. Wu et al., "Combining feature-level and decision-level fusion in a hierarchical classifier for emotion recognition in the wild," Journal on Multimodal User Interfaces, vol. 10, no. 2, pp. 125-137, 2016.

[9] T. Tuncer, S. Dogan, and A. Subasi, "A new fractal pattern feature generation function based emotion recognition method using EEG," Chaos, Solitons \& Fractals, vol. 144, pp. 110671-110675, 2021. 
[10] Y. Fang, H. Yang, X. Zhang, H. Liu, and B. Tao, "Multi-feature input deep forest for EEG-based emotion recognition," Frontiers in Neurorobotics, vol. 2, pp. 14-15, 2021.

[11] K. Zvarevashe and O. O. Olugbara, "Recognition of speech emotion using custom 2D-convolution neural network deep learning algorithm," Intelligent Data Analysis, vol. 24, no. 5, pp. 1065-1086, 2020.

[12] M. Zhang and L. Zhang, "Cross-cultural O2O English teaching based on AI emotion recognition and neural network algorithm," Journal of Intelligent and Fuzzy Systems, vol. 11, pp. 1-12, 2020.

[13] S. R. Bandela and T. K. Kumar, "Speech emotion recognition using unsupervised feature selection algorithms," Radioengineering, vol. 29, no. 2, pp. 353-364, 2020.

[14] K. M. Puk, S. Wang, J. Rosenberger et al., "Emotion recognition and analysis using ADMM-based sparse group lasso," IEEE Transactions on Affective Computing, vol. 1, p. 1, 2019.

[15] Y. Shi, Z. Chen, Z. Qi, and F. Meng, "A novel clustering-based image segmentation via density peaks algorithm with midlevel feature," Neural Computing \& Applications, vol. 28, no. 1, pp. 29-39, 2017.

[16] X. Xu, T. Jia, Q. Li, F. Wei, L. Ye, and X. Wu, "EEG feature selection via global redundancy minimization for emotion recognition," IEEE Transactions on Affective Computing, vol. 1, p. 1, 2021.

[17] B. Mocanu, R. Tapu, and T. Zaharia, "Utterance level feature aggregation with deep metric learning for speech emotion recognition," Sensors, vol. 21, no. 12, pp. 4233-4238, 2021.

[18] H. He, Y. Tan, J. Ying, and W. Zhang, "Strengthen EEG-based emotion recognition using firefly integrated optimization algorithm," Applied Soft Computing, vol. 94, pp. 106426106429, 2020.

[19] Y. Yang, M. Li, and X. Ma, "A point cloud simplification method based on modified fuzzy C-means clustering algorithm with feature information reserved," Mathematical Problems in Engineering, vol. 2020, no. 4, 13 pages, Article ID 5713137, 2020.

[20] Y. Zhang, X. Ji, and S. Zhang, "An approach to EEG-based emotion recognition using combined feature extraction method," Neuroscience Letters, vol. 633, pp. 152-157, 2016.

[21] L. Y. Mano, A. Mazzo, J. R. T. Neto et al., "Using emotion recognition to assess simulation-based learning," Nurse Education in Practice, vol. 36, pp. 13-19, 2019.

[22] K. Yea-Hoon, S. Sae-Byuk, and K. Shin-Dug, "Electroencephalography based fusion two-dimensional (2d)-convolution neural networks (CNN) model for emotion recognition system," Sensors, vol. 18, no. 5, p. 1383, 2018.

[23] J. Kacur, B. Puterka, J. Pavlovicova, and M. Oravec, "On the speech properties and feature extraction methods in speech emotion recognition," Sensors, vol. 21, no. 5, p. 1888, 2021.

[24] L. Schaan, A. Schulz, S. Nuraydin et al., "Interoceptive accuracy, emotion recognition, and emotion regulation in preschool children," International Journal of Psychophysiology, vol. 138, pp. 47-56, 2019.

[25] T. Özseven, "A novel feature selection method for speech emotion recognition," Applied Acoustics, vol. 146, pp. 320326, 2019. 